\title{
A Educação em saúde no pré-natal: perspectivas e realidade sob o olhar acadêmico
}

Health education in prenatal care: perspectives and reality from the academic point of view

Educación para la salud en el prenatal: perspectivas y realidad desde el punto de vista académico

Marcela Godinho Miranda do Vale ${ }^{1 *}$, Renato da Costa Teixeira ${ }^{1}$, John Henry de Oliveira Vale ${ }^{2}$, Jackson Celso Pereira Pires².

\section{RESUMO}

Objetivo: Compreender a percepção dos discentes de Fisioterapia de um curso de uma universidade pública no interior do estado do Pará sobre a educação em saúde na assistência pré-natal. Métodos: Trata-se de uma pesquisa de campo, de caráter qualitativo, do tipo descritiva, a qual a coleta de dados se deu através da técnica grupo focal, onde a amostra foi dividida em dois grupos com 6 e 9 participantes. Resultados: Após a análise dos dados no software Iramuteq foram grados três grupos textuais com os temas: Vivência de Educação em saúde no pré-natal, Quebra de paradigma e sua importância, e Recursos utilizados para educar em Saúde. A partir dos quais foi possível analisar a percepção dos discentes acerca da importância de atuar com Educação em saúde, a formação acadêmica e a quebra de paradigma para atuar na educação em saúde e recursos utilizados para educar em saúde. Conclusão: Foi constatado que os discentes que participaram da pesquisa não passaram por um processo gradativo de aprendizado para realizar as práticas de educação em saúde. No entanto, ao final das experiências se sentiam seguros e realizavam as atividades com uso de materiais que os próprios construíram e que executavam as ações com tranquilidade.

Palavras-chave Educação em saúde, Pré-natal, Alunos.

\begin{abstract}
Objective: To understand the perception of Physiotherapy students in a course at a public university in the interior of the state of Pará about health education in prenatal care. Methods: This is a qualitative, descriptive field research, which data collection was done through the focal group technique, where the sample was divided into two groups with 6 and 9 participants. Results: After analyzing the data in the Iramuteq software, three text groups were created with the themes: Experience of Health Education in prenatal care, Breaking of paradigm and its importance, and Resources used to educate in Health. From which it was possible to analyze the students' perception about the importance of working with health education, academic training and breaking the paradigm to work in health education and resources used to educate in health. Conclusion: It was found that the students who participated in the research did not undergo a gradual learning process to carry out health education practices. However, at the end of the experiments, they felt safe and carried out the activities using materials that they themselves built and that carried out the actions with ease.
\end{abstract}

Keywords: Health education, Prenatal care, Students.

1 Universidade do Estado do Pará (UEPA), Belém - PA. *E-mail: m_godinho_m@yahoo.com.br

2 Universidade do Estado do Pará (UEPA), Santarém - PA. 


\section{RESUMEN}

Objetivo: Comprender la percepción de estudiantes de Fisioterapia de un curso de una universidad pública del interior del estado de Pará sobre la educación para la salud en la atención prenatal. Métodos: Se trata de una investigación de campo cualitativa, descriptiva, cuya recolección de datos se realizó mediante la técnica de grupo focal, donde la muestra se dividió en dos grupos de 6 y 9 participantes. Resultados: Luego de analizar los datos en el software Iramuteq, se crearon tres grupos de texto con los temas: Experiencia de Educación en Salud en la atención prenatal, Rompiendo paradigma y su importancia, y Recursos utilizados para educar en Salud. A partir de los cuales se pudo analizar La percepción de los estudiantes sobre la importancia de trabajar la educación para la salud, la formación académica y romper el paradigma para trabajar en la educación para la salud y los recursos utilizados para educar en salud. Conclusión: Se encontró que los estudiantes que participaron en la investigación no pasaron por un proceso de aprendizaje gradual para realizar prácticas de educación en salud. Sin embargo, al finalizar los experimentos, se sintieron seguros y realizaron las actividades utilizando materiales que ellos mismos construyeron y que realizaron las acciones con facilidad.

Palabras clave: Educación para la salud, Atención prenatal, Estudiantes.

\section{INTRODUÇÃO}

Historicamente, no Brasil, a educação em saúde tem início no final do século XIX e começo do século XX. Ela está presente em todos os cenários que abrangem a vida do ser humano, promovendo interação entre as pessoas envolvidas dentro do contexto educativo e destas com a realidade em que vivem, visando à modificação de ambas as partes (BROLINI G, 2013; VASCONCELOS EM, 2001).

Consequentemente tem importante função no fortalecimento e qualidade de saúde do ser humano, pois através de suas práticas, sejam elas pedagógicas ou participativas, englobam saberes que compreendem os diversos campos de atuação, empoderam os indivíduos e as comunidades a desenvolverem suas capacidades, obtendo a clara reflexão crítica sobre a realidade que vivem (LIMA GCBB, et al., 2019).

No Brasil, as estratégias de educação em saúde na assistência pré-natal, de maneira individual ou em grupos educativos, são políticas de saúde que tem sido adotada pelo Ministério da Saúde e pelos governos, nas esferas municipais e estaduais (REBERTE LM, 2012).

Entre elas estão as diretrizes propostas pelo Programa de Atenção Integral à Saúde da Mulher e o Programa de Humanização do Pré-natal ao Nascimento, ambos instituídos pelo Ministério da Saúde em 1984 e 2000, respectivamente. Estes retratam que as ações educativas devem ser desenvolvidas por todos os profissionais que integram a equipe da unidade de saúde, com objetivo de levar a população a refletir sobre sua saúde, a adotar práticas para a sua manutenção, a realizar mudanças e incorporar novos hábitos para solução e melhoria de seus problemas de saúde (REBERTE LM, 2012).

Ao avaliar as concepções dos profissionais de saúde a respeito da educação em saúde, é possível compreender a relação desses profissionais com seu trabalho, sua percepção do processo educativo no cuidado e assistência ao usuário e, o modo como o profissional interpreta as ações de educação em saúde, podendo assim, estabelecer o seu perfil de atuação na sua prática (VIEIRA MSN, 2014).

$\mathrm{Na}$ tentativa de romper com o paradigma de formação baseada em um modelo hospitalocêntrico e biomédico, foram aprovadas em 2002 as Diretrizes Curriculares Nacionais (DCN's) para as graduações da área da saúde que refletem, a formação do profissional de saúde deve estimular o pensamento crítico e reflexivo, ser amparada na realidade da população e valorizar a produção do conhecimento nas comunidades e unidades de saúde, fazendo o alinhamento com as ciências produzidas nas academias (GONÇALVES FGG, et al., 2012).

Contribuindo para uma transformação nos cursos da área da saúde, as DCN's geraram mudanças nos projetos político-pedagógicos, que se readequaram às necessidades do Sistema Único de Saúde (SUS). 
Partindo do ideal que o SUS seja um grande ordenador de trabalho para os profissionais da saúde, merece destaque a preocupação com a formação dos profissionais da Fisioterapia, como estão sendo preparados para atuar de forma a promover a saúde através da educação, umas vez que as transformações políticas, ideológicas e organizativas que aconteceram no setor da saúde foram acompanhados tardiamente pela profissão que remonta um caráter reabilitador e o SUS apresenta-se com diretrizes predominantemente preventivas (SILVA CVP e ALMEIDA MMC, 2016).

Em Santarém, um dos locais que realiza assistência pré-natal de alto risco é o Centro de Referência em Saúde da Mulher, fundado em 2001, atendendo gestantes de todo município de Santarém. Como centro de referência, sempre é utilizado como campo de estágio dos diversos cursos da área da saúde, dentre eles, discentes do curso de Fisioterapia da Universidade do Estado do Pará (BRASIL, 2021).

Diante do exposto, este estudo tem como objetivo verificar qual a percepção dos acadêmicos de fisioterapia sobre a educação em saúde na assistência pré-natal em uma universidade pública no interior do estado do Pará.

\section{MÉTODOS}

Trata-se de uma pesquisa de campo, de caráter qualitativo, do tipo descritiva e transversal. Foi utilizado o processo de aplicação de entrevistas coletivas, utilizando-se da técnica de grupos focais para coleta de dados, opiniões, vivências e conceitos de discentes de Fisioterapia em uma universidade pública no interior do estado do Pará, sobre a atuação do fisioterapeuta em Educação em Saúde na assistência pré-natal. A amostra foi composta por 15 (quinze) discentes, estes foram divididos em dois grupos (Grupo 1 - 6 discentes e Grupo 2 - 9 discentes) de acordo com as normas da técnica grupo focal que foi escolhida para coleta de dados da pesquisa.

Foram coletados dados demográficos durante o mês de julho de 2018. Em sendo, apresentar os seguintes critérios de inclusão: estar regularmente matriculado na instituição de ensino superior (IES); cursar o $4^{\circ}$ ou $5^{\circ}$ ano do curso de Fisioterapia; ter realizado a disciplina de estágio curricular de Fisioterapia em Saúde da Mulher em um centro de saúde especializado, assinar o termo de consentimento livre e esclarecido (TCLE). Critérios de exclusão: ter realizado atividades de educação em saúde no CRSM através de outros projetos de iniciação científica.

Assim, foi seguida uma linha metodológica de acordo com a sequência: planejamento, condução das sessões e coleta dos dados. A fim de possibilitar um agrupamento de temas e a categorização do material discursivo, sendo utilizado um roteiro semiestruturado, que foi utilizado apenas para nortear o encontro. As regras de condução das sessões foram pactuadas no grupo, para que os participantes se sentissem à vontade para se manifestar, quais foram: não interromper a fala dos colegas, não exaltar o tom de voz, não contestar a fala do outro com palavras agressivas e respeitar a opinião ouvida.

As falas dos participantes foram gravadas através de um aplicativo de áudio e posteriormente armazenadas em um notebook. Para apoiar a análise dos dados desta pesquisa, foi utilizado o software IRAMUTEQ®. A análise interpretativa do conteúdo obtido se deu pela Análise de Conteúdo, considerando o conjunto de características em um determinado fragmento do conteúdo, gerado pela análise do Iramuteq ${ }^{\circledR}$ (BARDIN L, 2011). Após a conclusão destas etapas, segue-se a fase final da análise, composta pela apresentação dos resultados de forma descritiva e ilustrativa das falas, acompanhada da discussão, interpretação do material (CAMARGO BV e JUSTO AM, 2010; MINAYO MCS, 2010 e TURATO ER, 2013).

Esta pesquisa obteve parecer favorável pelo Comitê de Ética em Pesquisa da Universidade do Estado do Pará, Campus XII - Tapajós, com o número de registro CAAE 80736117.8.0000.5168. A sua coleta de dados foi codificada para garantir o anonimato dos participantes, que assinaram o Termo de Consentimento Livre e Esclarecido (TCLE), que está de acordo com a Resolução 466/12 do Conselho Nacional de Saúde (CNS), conforme as normas de realização de pesquisas que envolvem seres humanos. 


\section{RESULTADOS}

O texto obtido foi utilizado para análise através do programa Iramuteq pelo método de Reinert onde foi possível a análise em três grupos textuais gerados de acordo com as perguntas norteadoras elaboradas pela autora. O grupo1 com o tema Vivência de Educação em saúde no pré-natal, onde estavam agrupadas as respostas relacionadas as questões norteadoras 1 e 2 ( $O$ que você poderia dizer sobre a Educação em Saúde na assistência pré-natal de alto risco? / Como o fisioterapeuta pode atuar na Educação em Saúde?) utilizadas no grupo focal. O grupo 2 com o tema Quebra de paradigma e sua importância, onde estavam agrupadas as respostas relacionadas as questões norteadoras 3 e 4 (Como foi/está sendo a sua formação para o trabalho na assistência pré-natal de alto risco através da Educação em saúde? / Qual a importância da Educação em Saúde no processo de ruptura dos paradigmas tradicionais de formação do fisioterapeuta?).

Através desses grupos textuais formados foi possível gerar três categorias de análises após a leitura dos corpus textuais coloridos de cada grupo, são eles: Percepção de discentes sobre a importância da Educação em Saúde no pré-natal (Categoria 1); Formação Acadêmica e a quebra de paradigma para atuar na educação em saúde (Categoria 2); e Recursos utilizados para executar ações de educação em saúde (Categoria 3), representados abaixo.

\section{Categoria 1: Percepção de discentes sobre a importância da educação em saúde no pré-natal}

Nessa categoria foi possível abordar a percepção dos discentes sobre a importância da educação em saúde e das formas como são realizadas, com objetivo de perpetuação de conhecimento através das pessoas que estão recebendo a informação. Na visão dos discentes para que elas repassem para familiares e para sua comunidade, através de uma prática dinâmica e atrativa, como evidenciado nas falas abaixo:

"...tão legal assim você demonstrando de forma dinâmica para ela, porque uma coisa assim que para gente já é normal, que a gente já conhece mas a forma como ela recebeu a informação..." (Discente 3).

“...a gente já tá passando uma informação ela vai chegar lá na comunidade dela e vai falar para outras pessoas que ela atendeu porque eu acho que é uma semente que a gente planta..." (Discente 5).

Foi exposto pelos discentes a abordagem dos temas utilizados durante a sua experiência com a educação em saúde no pré-natal, temas como a importância da prevenção de complicações como hipertensão e diabetes; orientações para acelerar o trabalho de parto e a importância do fisioterapeuta no ambiente hospitalar/sala de parto; preparação para o parto durante o pré-natal com orientações de exercícios respiratórios e posições para amenizar as dores e acelerar o trabalho de parto; cuidados com o bebê e sua alimentação no pós - parto (amamentação exclusiva, armazenamento de leite e introdução alimentar), como demonstrado em algumas falas:

"...um fator de risco muito grande na hora do parto para de mortalidade tanto para a mãe quanto para o bebê e que as vezes a gente acha que já tá muito batido..." (Discente 6).

“...isso serviu muito porque muitas mães ali trabalhavam e tinham preocupação de retornar ao seu trabalho e não queriam introduzir o leite artificial..." (Discente 1).

Os discentes chegaram à conclusão de que assuntos aparentemente básicos e fáceis para eles, eram vistos como algo complexo pelo público que recebia a educação em saúde. Reforçando a necessidade da realização dessas práticas:

"...informações que pra gente parece ser muita besteira como por exemplo o fato de amamentar a criança até os seis meses exclusivamente..." (Discente 6).

“...tanto que eu lembro que uma...feijão para uma criança de 4 meses...” “...então que para a gente parece ser muito muito simples e muito óbvio para elas não é..." (Discente 9). 
A percepção dos discentes sobre a importância da inserção do Fisioterapeuta em campos desconhecidos pelos próprios profissionais de saúde como na promoção e prevenção no pré-natal com temas muitas vezes considerados repetitivos, porém na visão deles valorizados pelo público-alvo, em ambientes diferentes, como mostram as falas:

"...ela não sabia que a Fisioterapia poderia estar atuando naquilo então foi bem satisfatório e muito importante porque a maioria delas vivem em comunidades e não tem acesso..." (Discente 15).

A percepção do estranhamento de ter o Fisioterapeuta em ambientes do pré-natal e parto trabalhando diferentes temas e dar orientações que para quem trabalha na área da saúde pode ser simples, porém é importante tanto para gravidez atual, tanto para uma eventual próxima gravidez, como demonstram as falas:

"...quando a gente fala que é acadêmico de Fisioterapia nesse primeiro contato de educação em saúde da mulher muitas delas nem sabem que a gente pode auxiliar na hora do parto e também no pré-natal, então elas ficam bastante surpresas..." (Discente 13).

\section{Categoria 2: Formação acadêmica e a quebra de paradigma para atuar na educação em saúde}

Nessa categoria foi possível abordar a Inserção do Fisioterapeuta na Educação em saúde. Sob a ótica discente a importância de trabalhar de forma ética, atendendo as expectativas das pacientes, usando linguagem acessível, trabalhando desde orientação individual, durante a própria consulta, como o trabalho nos grupos de palestras ou rodas de conversas. Com intuito de trabalhar na prevenção quebrando a ideia do Fisioterapeuta ligado apenas a reabilitação, como mostram as falas:

“...então passar essas informações de educação ali para aquele paciente durante 0 atendimento perguntar se ele tem alguma dúvida e conversar..." (Discente 3).

“...estar bastante preparado não somente conhecimento, mas com os dispositivos, usar para atingir essas mães, deve ter conhecimento da população que ele vai atingir..." (Discente 7).

Foi possível refletir sobre a Educação em saúde e a prática acadêmica, os estudantes apontaram falhas da academia em ensinar a educar em saúde. Relatam que aprenderam com a prática e melhoraram a cada experiência, não sabiam como fazer, como escolher, o que falar e sentiam vergonha pelo que estavam fazendo, como falaram:

“...no início a gente parecia um bando de robô conforme a prática gente ia se soltando atrair mais público eu acho que a uepa pecou nisso que foi um pouco de susto e vai..." (Discente 4).

Os discentes relataram o contato tardio com ações na atenção primária em saúde, especificamente com a Educação em saúde:

“...pensado em fazer então eu acho que a gente se a gente pudesse já começar desde o primeiro ano com educação em saúde de seria ótimo..." (Discente 15).

Foi possível refletir sobre o desafio de aprender a Educar em saúde. Apesar das ditas falhas da academia, gostaram e superaram os desafios, viram a importância do que estavam fazendo, como falaram:

“...então eu acho que foi uma falha mas nem por isso a gente deixou de fazer a gente foi aprendendo com as críticas realmente quando depois que a gente terminar de fazer a pessoa e ela dizer olha..." (Discente 7).

“...a gente só consegue alguma coisa conhecimento passa através de algum desafio e por isso que surgiram as metodologias ativas na academia a nossa graduação foi a metodologia antiga tradicional desde o primeiro ano..."

(Discente 12). 


\title{
Categoria 3: Recursos utilizados para executar as ações de educação em saúde
}

Nessa categoria foi possível identificar as ferramentas utilizadas pelos discentes para realizar as ações de educação em saúde, assim como observar as dinâmicas executadas. Ensinando sobre assuntos considerados tabus e que são de grandes benefícios para as gestantes, como a incontinência urinária e posições para acelerar o trabalho de parto, como mostram as falas:

\begin{abstract}
“...uma incontinência urinária nessa mulher, nas nossas palestras nós sempre nos trazíamos impressa imagem da musculatura do assoalho pélvico, explicávamos que aquilo era feito de músculo que a mulher ela nem imaginava..." (Discente 5).
\end{abstract}

A utilização de material de baixo custo para educar em saúde. Confecção e utilização de materiais dos próprios discentes que tornavam a atividade mais dinâmica e fácil de ser compreendida pelo público. $\mathrm{A}$ criatividade e a empatia como meios de educar em saúde, como mostram as falas:

\begin{abstract}
"...o nosso grupo ele foi o grupo que confeccionou a reprodução do seio artificial e na divisão de materiais de baixo custo com isopor e tecido para realizar a confecção desse material..." (Discente 8).

“...nosso grupo a gente utilizou teve uma vez que a gente fez uma televisão nós colocamos pontos o que a gente queria falar que no caso era sobre a hipertensão arterial na gravidez e a diabetes gestacional..." (Discente 1).
\end{abstract}

\section{DISCUSSÃO}

A educação em saúde é definida como um conjunto de práticas participativas, que utiliza de dispositivos metodológicos para a promoção de mudança de estilo de vida e comportamento. Conglomerando saberes que envolvem os diversos campos de ação e que empoderam os indivíduos e a comunidade a desenvolverem suas competências como resposta de uma práxis baseada em reflexão crítica sobre a realidade. Através de tecnologias contemporâneas, é praticada com a expectativa de progresso da assistência aos indivíduos que recebem essa prática (LIMA GCBB, et al., 2019).

Confirmando a percepção da importância da realização de práticas que promovam e previnam em saúde, promovendo a autonomia no cuidado próprio e do recém-nascido no caso da realização da educação em saúde durante o período pré-natal, o estudo de Pio DAM e Oliveira MM (2014) também acredita na potência da educação em saúde, desde que voltada para o empoderamento social e não renegue as relações de poder envolvidas. Desta forma, ela pode produzir um saber coletivo que instigue nos indivíduos sua autonomia e emancipação para o cuidado de si, dos seus familiares/amigos e de sua realidade.

Para Falkenberg MB, et al. (2014) as práticas de educação em saúde envolvem três segmentos de atores prioritários: os profissionais de saúde que valorizem a prevenção e a promoção tanto quanto as práticas curativas; os gestores que apoiem esses profissionais; e a população que necessita construir seus conhecimentos e aumentar sua autonomia nos cuidados, individual e coletivamente.

Em um estudo realizado com Residentes, dentre eles um fisioterapeuta, na atenção básica possibilitou repensar sobre a prática profissional atual, baseada apenas na clínica tradicional, nos remetendo para além do individual através de um modelo assistencial fundamentado na multidisciplinaridade, integralidade e na humanização do cuidado, demonstrando que é possível a ampliação do trabalho voltado para a complexidade do processo gravídico e puerperal (LIMA JC, et al., 2014).

Ribeiro SB, et al. (2015) concluem em seu estudo que a inserção do fisioterapeuta como educador em saúde é um processo em construção e tem ocorrido de forma gradativa. Aos poucos se percebe que, por meio de sua atuação, pode-se reduzir a demanda de atendimentos e melhorar a qualidade de vida da população. 
Nota-se então que apesar de todos os entraves os estudantes da presente pesquisa se encontram atentos quanto a importância da atuação do fisioterapeuta na educação em saúde, neste caso, voltados para assistência pré-natal. Todos relataram ter tido mais de uma experiência com educação em saúde no pré-natal, com temas distintos e com boa aceitação do público-alvo, apesar de relatarem o estranhamento sobre a atuação do fisioterapeuta nesta área pelo próprio público.

A exemplo da Educação em saúde Mattos MP, et al. (2017) mostrou ao público em seu estudo que o fisioterapeuta ajuda a prevenir e tratar disfunções musculoesqueléticas que podem se instalar devido à má postura adotada pelas mães. Com base nessa discussão, destacou o papel do fisioterapeuta no processo de aleitamento materno, garantindo que as orientações e apoio desse profissional contribuem para fazer a diferença durante a amamentação, trazendo um maior conforto para a mãe e o bebê, chamando atenção para os aspectos de promoção, prevenção e recuperação da saúde.

Assis TR, et al. (2016) em seu estudo os estudantes de Fisioterapia participantes do projeto, tiveram oportunidade de ampliar seus conhecimentos, atuando na atenção básica, no âmbito da prevenção primária e em contato direto com o ser humano. Relata a importância de experiências no sentido de uma mudança na concepção de saúde e na perspectiva de atuação dos estudantes e futuros fisioterapeutas. Considerando ser o fisioterapeuta um profissional de saúde que tem a formação excessivamente voltada para a reabilitação, é muito enriquecedora a oportunidade de acompanhar as condições de vida e de saúde das pessoas inseridas em sua realidade, desenvolvendo ações que promovam a saúde e contribuam com a assistência integral.

Diferente do discurso relatado pelos acadêmicos da UEPA, no estudo de Assis TR, et al. (2016) previamente ao início das atividades com as gestantes, os estudantes envolvidos realizaram uma capacitação com a professora coordenadora, em reuniões semanais, por um mês. Nesses encontros, foram simuladas as atividades, dinâmicas e conteúdo que seriam explorados nas oficinas, com o intuito de oferecer ao público-alvo o melhor atendimento e contato possível entre ensino e sociedade.

Concordando com a experiência relatada pelos discentes no atual estudo Lima JC, et al. (2014) afirmam que a educação em saúde não pode ocorrer como ação mecânica e automática, mas como uma ação planejada, dinâmica, sensível, múltipla e criativa, implicando vontade, ação, habilidades, técnica, ética, estética, que superam o conhecer, alegria, satisfação e prazer.

Para Seriano KN, et al. (2013) como forma de distanciar a Fisioterapia do paradigma de ser uma profissão apenas reabilitadora, a educação deve-se basear na comunidade, o que possibilita 0 desenvolvimento e treinamento de habilidades e competências como liderança, trabalho em equipe multiprofissional, interação com a comunidade, resolução de problemas, comunicação e planejamento buscando desta forma a integralidade do atendimento e a implantação real e efetiva dos princípios do SUS.

Apesar do fisioterapeuta ser reconhecido como profissional indispensável na área da saúde, este ainda é visto, muitas vezes, como reabilitador, mascarando com isso uma de suas funções principais, que é a atuação no campo preventivo e de promoção à saúde. A própria origem da Fisioterapia direcionou as definições do campo profissional para atividades reabilitadoras. No entanto, a formação universitária, como especificado pelo Ministério de Educação (MEC), destaca o fisioterapeuta como um profissional generalista, sendo capaz, portanto, de atuar em todos os níveis de atenção à saúde, não devendo ficar restrito às ações curativas e reabilitadoras (RIBEIRO SB, et al., 2015).

\section{CONCLUSÃO}

Neste estudo foi constatado que os discentes que participaram da pesquisa não passaram por um processo gradativo de aprendizado para realizar as práticas de educação em saúde. No entanto, ao final das experiências se sentiam seguros e realizavam as atividades com uso de materiais que os próprios construíram e que executavam as ações com tranquilidade. 


\section{REFERÊNCIAS}

1. ASSIS TR, et al. Oficinas para Gestantes nas Unidades Básicas de Saúde de Jataí-GO: Ações Integradoras de Saberes. Revista Interagir: pensando a extensão, Rio de Janeiro, 2016; 22: 174-178.

2. BARDIN L. Análise de conteúdo. Lisboa: Edições 70, 2011.

3. BISPO JÚNIOR JP. Fisioterapia e saúde coletiva: desafios e novas responsabilidades profissionais. Ciência \& Saúde Coletiva, 2010; 15(1): 1627-36.

4. BRASIL. Ministério da Saúde (MS). Secretaria de Gestão do Trabalho e da Educação na Saúde. Departamento de Gestão e da Regulação do Trabalho em Saúde. Câmara de Regulação do Trabalho em Saúde. Brasília: MS, 2006.

5. BRASIL. Ministério da Saúde (MS). Secretária de Atenção à Saúde. CNES Net. Estabelecimento de Saúde. Disponível http://cnes2.datasus.gov.br/Exibe Ficha Estabelecimento.asp?VCo Unidade $=1506802330172 \&$ VEstado $=15 \&$ VC odMunicipio=150680. Acesso em: 10/04/2021

6. BROLINI G. Educação em saúde no contexto da assistência ao estudante no Instituto Federal de Educação, Ciência e Tecnologia de Roraima. Dissertação (Mestrado Profissional) - Universidade Federal de São Paulo. São Paulo, 2013; 68 p.

7. CAMARGO BV, JUSTO AM. Tutorial para uso do software de análise textual IRAMUTEQ. Laboratório de psicologia Social da Comunicação e Cognição - LACCOS. Universidade Federal de Santa Catarina, Brasil 2013.

8. FALKENBERG MB, et al. Educação em saúde e educação na saúde: conceitos e implicações para a saúde coletiva. Revista Ciência \& Saúde Coletiva, 2014; 19(3): 847-852.

9. GONÇALVES FGG, CARVALHO BG, TRELHA CS. O ensino da Saúde Coletiva na Universidade Estadual de Londrina: da análise documental à percepção dos estudantes. Trabalho de Educação em Saúde, Rio de Janeiro, 2012; 10(2): 301-314.

10. LIMA JC, et al. Gestação Vida: oficina educacional para gestantes com abordagem multiprofissional em uma Unidade Básica de Saúde da cidade de Manaus. Revista Extensão em Foco, Curitiba: Editora da UFPR, 2014; 10: 86-101.

11. LIMA GCBB, GUIMARÃES AMDN, SILVA JRS, OTERO LM, GOIS CFL. Educação em saúde e dispositivos metodológicos aplicados na assistência ao Diabetes Mellitus. Saúde Debate, Rio de Janeiro, 2019; 43(120): 150158.

12. MACHADO MFAS, et al. Integralidade, formação de saúde, educação em saúde e as propostas do SUS - uma revisão conceitual. Revista Ciências \& Saúde Coletiva, 2007; 12(2): 335-342.

13. MATTOS MP, et al. Educação em saúde no processo de posicionamento da mãe com o bebê durante a amamentação. Revista Em Extensão, 2017; 16(2): 242-5.

14. MINAYO MCS. Pesquisa Social: teoria, método e criatividade. 29. ed. Petrópolis: Vozes, 2010.

15. PIO DAM, OLIVEIRA MM. Educação em saúde para atenção à gestante: paralelo de experiências entre Brasil e Portugal/Health education in pre-natal care: a parallel of experiences between Brazil and Portugal. Revista Saúde Soc. São Paulo, 2014; 23(1): 313-324.

16. REBERTE LM. Efeitos do uso de uma cartilha educativa durante o pré-natal. Tese (Doutorado) - Escola de Enfermagem da Universidade de São Paulo. São Paulo, 2012; 144 p.

17. RIBEIRO SB, et al. A Importância do Fisioterapeuta como Agente Educador de Saúde na Unidade Básica de Saúde da Família. Revista Brasileira de Saúde Funcional, 2015; 2(1): 05-14.

18. SERIANO KN, et al. Percepção de estudantes do curso de fisioterapia sobre sua formação profissional para atuação na atenção básica no Sistema Único de Saúde. Revista Fisioterapia Pesq; 2013; 20(3): 250-255.

19. SILVA CVP, ALMEIDA MMC. Fisioterapia e Saúde coletiva; uma análise crítica da produção científica no Brasil. Cadernos de Educação Saúde e Fisioterapia, 2016; 1(3): 33-40.

20. TURATO ER. Tratado de Metodologia da pesquisa clínico-qualitativa: construção teórico-epistemológica, discussão comparada e aplicação nas áreas da saúde e humanas. 6ª ed. Petrópolis: Editora Vozes, 2013.

21. VASCONCELOS EM. Participação popular e educação nos primórdios da saúde pública brasileira. In: VASCONCELOS EM. A saúde nas palavras e nos gestos: reflexões da Rede de Educação Popular nos Serviços de Saúde. São Paulo: Editora Hucitec, 2001.

22. VIEIRA MSN. Práticas de educação em saúde de nutricionistas da secretaria municipal de saúde de Goiânia. Dissertação (Mestrado) - Universidade Federal de Goiás, Faculdade de Medicina, Goiânia, 2014; 87p. 